\title{
Palmar Dermatoglyphics of Japanese Macaques, Macaca fuscata, in the Kyushu and Tohoku Districts, Japan
}

\author{
Mitsuo Iwamoto $^{1}$ and Bambang SuRyobroto ${ }^{2}$ \\ 1 Primate Research Institute, Kyoto University, \\ Inuyama, Aichi 484, Japan. \\ 2 Department of Biology, Bogor Agricultural University, \\ Jl. Raya Pajajaran, Bogor, Indonesia.
}

Received July 8, 1993

\begin{abstract}
The palmar dermatoglyphics of 461 Japanese macaques (Macaca fuscata) were studied. The sample was divided into five groups according to geographical location, and included four groups of the subspecies Macaca $f$. fuscata and one group of the subspecies $M . f$. yakui. Chi-square analysis of group differences in the frequencies of dermatoglyphic pattern type and ridge direction showed significant intraspecific differentiation. The two groups showing the most differences from the other three groups were the subspecies Macaca f. yakui (YAKUS group) and the northernmost group of $M . f$. fuscata (SIMOK group) from the Shimokita Peninsura. Cluster analysis applied to pattern intensity values, which were derived from the pattern types, confirmed the greater biological distance of the SIMOK and especially the YAKUS groups from the others. The extent of intraspecific variation observed in the dermatoglyphic system may reflect the operation of genetic drift in these insular populations.
\end{abstract}

Key Words: dermatoglyphics, palm, Japanese macaque, Macaca fuscata, subspecies

\section{INTRODUCTION}

In 1964, one of the present authors (Iwamoto, 1964) reported that the two subspecies of the Japanese macaque (Macaca fuscata fuscata and M. f. yakui) differed with respect to frequencies of palmar dermatoglyphic patterns and ridge direction. Subsequently, he also documented similar group differences in the dermatoglyphic features of the foot (Iwamoto, 1967). Iwamoto's conclusions were later confirmed by statistical analysis (Cauble and Mavalwala, 1973). The purpose of the present study is to expand the earlier work by (1) adding more materials collected from the same two groups studied previously and new materials from three groups of $M . f$. fuscata, two located in the Kyushu District of southern Japan and one group in the Tohoku District of northern Japan; and (2) statistically testing for inter-group differences and measuring group affinities within the species $M$. fuscata. 


\section{SAMPLES AND METHODS}

The dermatoglyphic data used in the present study came from the left and right palmar prints of 461 Japanese macaques. The total sample consisted of five groups identified by geographical location (Table 1, Fig. 1). Four groups (SIMOK, KAWAR, TAKAS, KOSIM) belong to the subspecies $M$. f. fuscata and one group (YAKUS), to the subspecies Macacaf. yakui.

The dermatoglyphic features were read and classified according the nomenclature given in Iwamoto and Suryobroto (1990), slightly simplified for purposes of the present study. Ridge direction (RD) is defined by the orientation of the proximal ridge line starting from interdigital area II or III, and the volar area where it terminates (Iwamoto and Suryobroto, 1990) (Fig. 2).

Group differences in frequencies of pattern type and ridge direction at each volar area were tested separately by side using chi-square analysis, and in the text, "***" indicates "significant at $P<0.01$ " for both sides.

For the evaluation of group affinities, a measure of pattern intensity was derived wherein values 0,1 and 2 were assigned to the pattern types $\mathrm{O}, \mathrm{L}$ and $\mathrm{W}$, respectively. Because chi-square testing had yielded no significant differences in pattern type by sex and side, the pattern intensity values were combined for group comparisons. The mean pattern intensity was computed by group for each of the seven palmar areas. From these values, a 5 (group) $\times 7$ (palmar area) matrix table was generated and used in the cluster analysis from which a cladogram was produced.

\section{RESULTS AND DISCUSSION}

Frequencies of pattern type and ridge direction at each volar area were given in Tables 2 and 3. The sexes and sides were combined, because chi-square testing had yielded no significant differences in pattern type ridge and direction by sex and side.

Thenar pattern (Th): The patterns of thenar area are relatively simple, usually being type $\mathrm{O}$ or type $\mathrm{L}$ (Lu with the exception of $\mathrm{Lr}$ in two palms of TAKAS group). SIMOK and YAKUS groups were similar in their high frequencies of type $\mathrm{L}(89$ and $78 \%)$, differing significantly $(* *)$ from the lower frequencies $(33-40 \%)$ exhibited by the other three groups.

The first interdigital pattern (I): The first interdigital pattern is predominantly type $\mathrm{L}$ (Ld in all palms) followed by type W (Wr in most palms, Wo in some) or type O. The TAKAS group shows a relatively high frequency of type W (30\%), differing significantly $(* *)$ from the lower frequencies $(<8 \%)$ characteristic of the other three groups except YAKUS group (19\%).

The second interdigital pattern (II): The second interdigital pattern is usually type $\mathrm{W}$ (various frequencies of $\mathrm{Wu}$, Wo and $\mathrm{Wr}$ ) and sometimes type $\mathrm{L}$ ( $\mathrm{Ld}$ or $\mathrm{Lp}$ ). 
Table 1. The group name and the total number of animals studied

\begin{tabular}{lcll}
\hline Group & Number & \multicolumn{1}{c}{ Location } & Prefecture \\
\hline SIMOK & 35 & Shimokita Peninsula & Aomori \\
KAWAR & 53 & Mt. Kawaradake & Fukuoka \\
TAKAS & $118^{1)}$ & Mt. Takasaki & Oita \\
KOSIM & 117 & Koshima Islet & Miyazaki \\
YAKUS & $138^{2)}$ & Yaku Island & Kagoshima
\end{tabular}

1) Includes 56 animals reported previously by Iwamoto (1964).

2) Includes 40 animals reported previously by Iwamoto (1964).

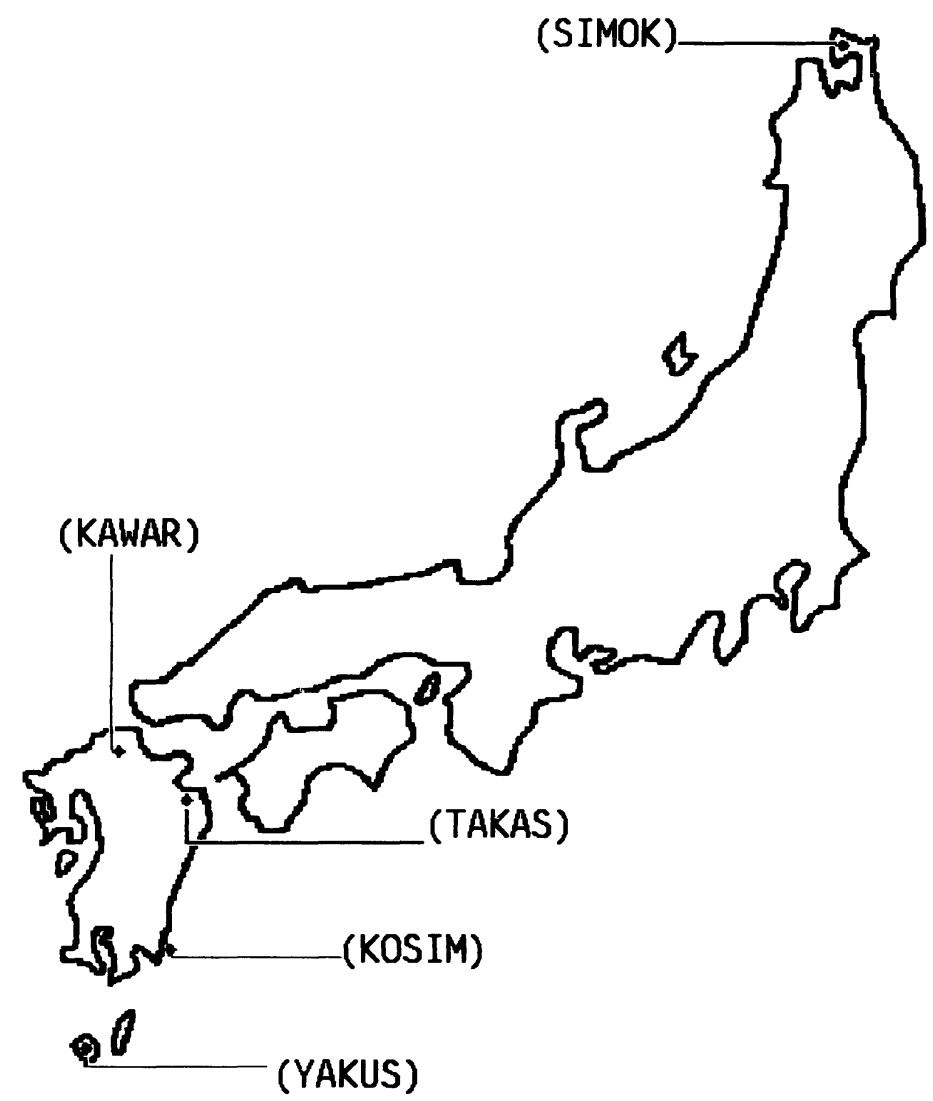

Fig. 1. Localities of the groups examined. Group names corresponding to those of Table 1 are given in parentheses.

The SIMOK group was unique in its significantly $(* *)$ higher frequency of type L $(21 \%)$ when compared to the remaining group $(<6 \%)$.

The third interdigital pattern (III): The third interdigital pattern is usually type $\mathrm{W}$ (various frequencies of $\mathrm{Wr}, \mathrm{Wo}$ and $\mathrm{Wu}$ ) or $\mathrm{L}$ (mainly Ld, sometimes $\mathrm{Lp}$ ). The 

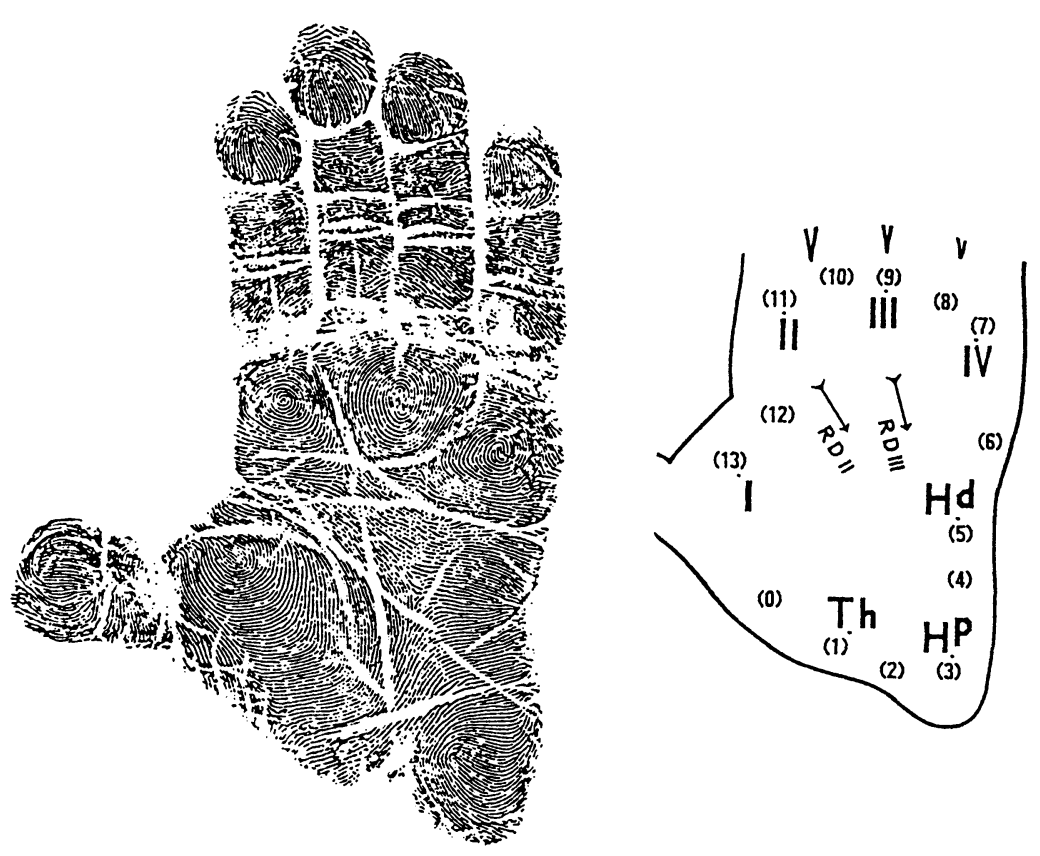

Fig. 2. Dermatoglyphic print of the hand from a macaque (left) and distinction of the palmar dermatoglyphic areas and patterns (right). (From Iwamoto and Suryobroto, 1990)

KOSIM group shows a relatively high frequency of type L (49\%), differing significantly $(* *)$ from the other three groups $(<20 \%)$ except KAWAR group $(38 \%)$.

The fourth interdigital pattern (IV): The fourth interdigital pattern is usually type $\mathrm{W}$ (various frequencies of $\mathrm{Wr}$, Wo and $\mathrm{Wu}$ ) and occasionally type $\mathrm{L}$ ( $\mathrm{Lu}$ or $\mathrm{Ld}$ ). No significant differences were found, with the exception of the few detected in comparison of the right hand.

Distal hypothenar pattern $\left(\mathrm{H}^{\mathrm{d}}\right)$ : The YAKUS group differed significantly $(* *)$ in its fixation of type $\mathrm{O}$, from the other four groups in which $\mathrm{L}$ type also occurred frequently.

Proximal hypothenar pattern $\left(\mathrm{H}^{\mathrm{p}}\right)$ : The types $\mathrm{L}$ and $\mathrm{W}$ were the only patterns at the proximal hypothenar area. Again, the YAKUS group approached fixation of type L in this area (97\%), differing significantly $(* *)$ from the other three groups $(<48 \%)$. The SIMOK group also differed significantly (**) from all others, but in this case, approached fixation of type W (99\%).

Ridge Direction II (RD II): The SIMOK and YAKUS groups were similar in RD II with a predominantly ulnar orientation of the ridges (100 and 93\%), which usually terminated in areas $4-6$. This orientation differs significantly $(* *)$ from the other three groups in which the ridge line is usually oriented proximally and terminates in areas 1-2 (usually in 2) (83-91\%). 
Palmar Dermatoglyphics of Japanese Macaques

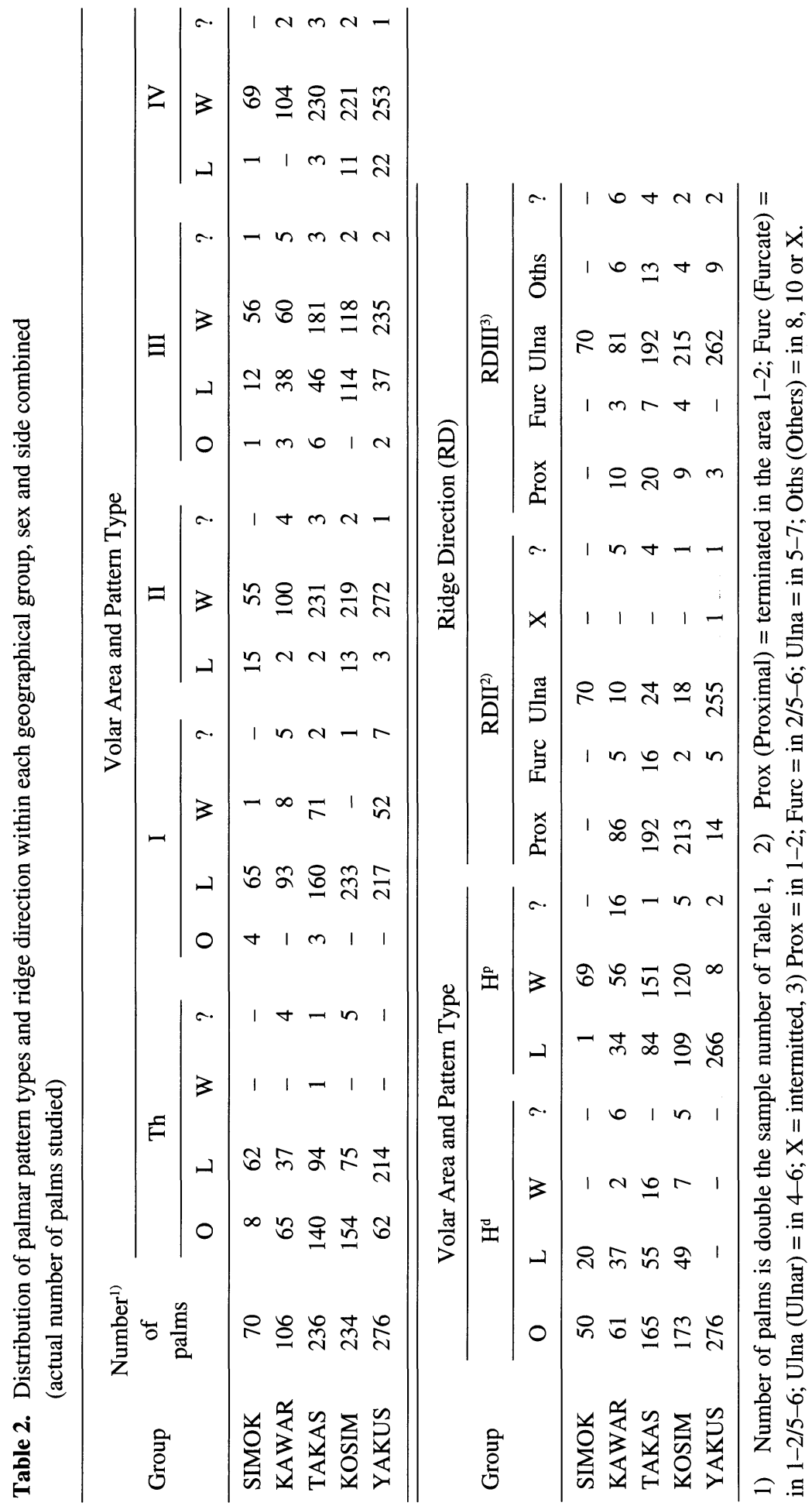




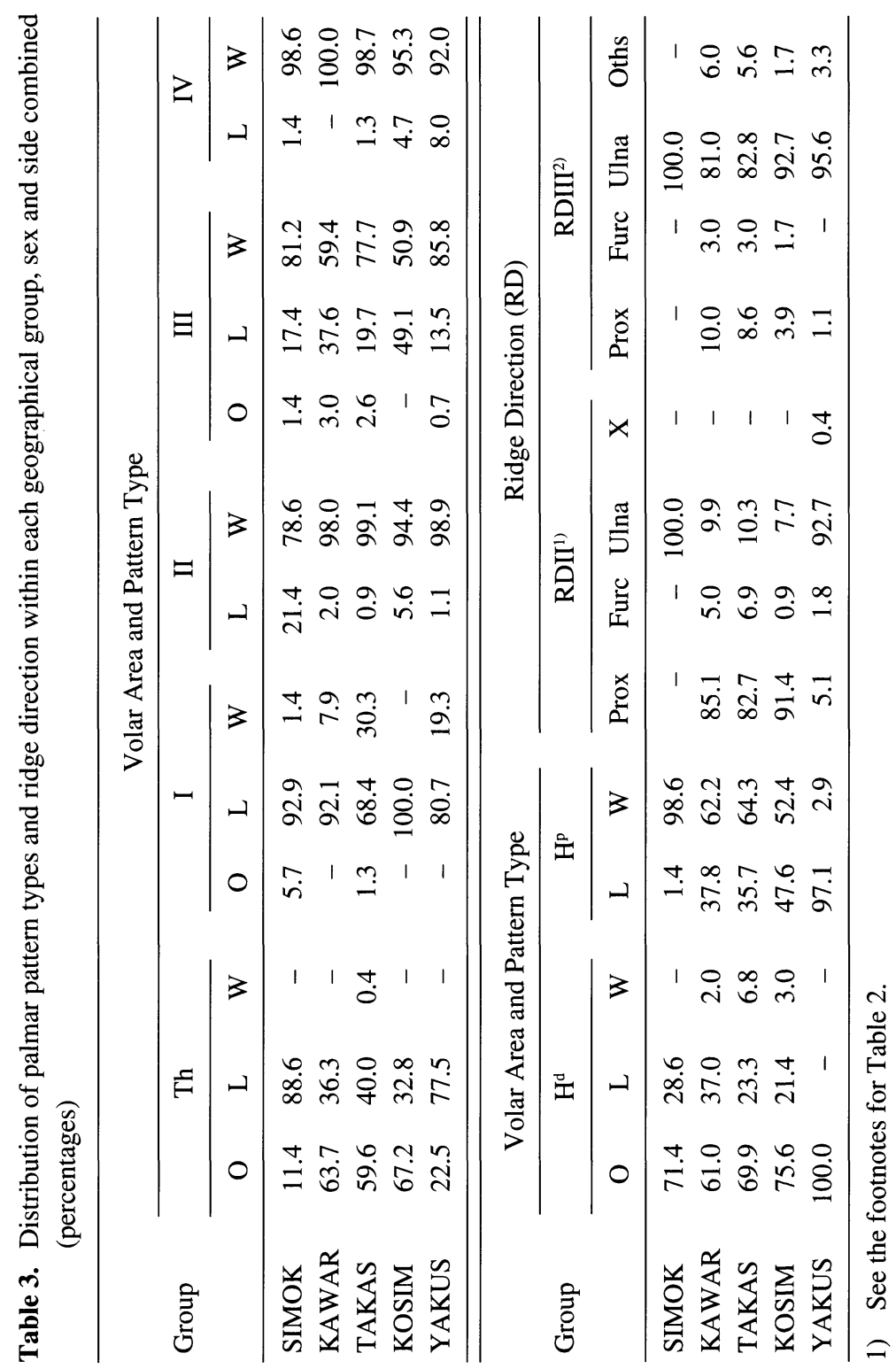


Ridge Direction III (RD III): RD III was almost exclusively ulnar in direction and terminated in areas 5-7 (usually in 6) in all groups (81-100\%) with no significant group differences.

Cluster analysis, based on pattern intensity, revealed that the YAKUS group diverged from the other four groups (Fig. 3). The separation was primarily attributable to the combined higher frequencies of type L (78-97\%) at both the thenar and proximal hypothenar areas and type $\mathrm{O}(100 \%)$ at the distal hypothenar area which characterized the YAKUS group. Of the remaining four groups, SIMOK group resembled the YAKUS in the high frequency of type $\mathrm{L}$ at the thenar area (89\%). At the proximal part of hypothenar area, however, the dominant type for the SIMOK group was type $\mathrm{W}(99 \%)$, rather than type L (1\%). Thus, in the cluster analysis SIMOK group attained an intermediate position between YAKUS group and the cluster composed of the KAWAR, TAKAS and KOSIM groups.

The significant differences between each of SIMOK and YAKUS groups and each of the other three groups, KAWAR, TAKAS and KOSIM, in ridge direction II (RD II) also suggest that the latter three groups constitute one cluster.

The reaults presented here confirm that there are significant inter-group differences in dermatoglyphic features of the palm, not only between the separate subspecies of Macaca fuscata, but also between several groups of the subspecies Macaca f. fuscata resident in different locales. The southernmost group, YAKUS of the subspecies Macaca f. yakui exhibited the greatest biological distance from the other four groups, all of which are members of the subspecies $M$. f. fuscata. YAKUS is the southernmost group, isolated on Yaku-shima Island from the other southern groups.

Significant, albeit reduced, dermatoglyphic differences were also found among the remaining four groups, all of which belong to the same subspecies. Here the most divergent group was SIMOK, which lives on the northern penninsula of Shimokita, isolated from the three groups all of which reside in the Kyushu district in southern Japan.

The extent of intraspecific variation observed here in the dermatoglyphic system may reflect the operation of genetic drift in these insular populations.

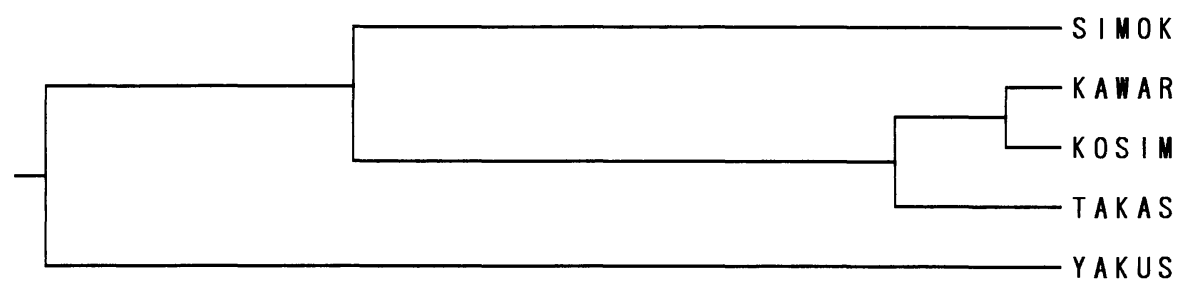

Fig. 3. Group affinities for five groups of Japanese macaques based on clustering analysis applied to dermatoglyphic pattern intensity of the palm. 


\section{ACKNOWLEDGEMENTS}

We thank the many individuals who assisted us in collecting the data for this study, including Yuzuru Hamada and Tsuyoshi Watanabe. We thank Laura NewellMorris, University of Washington, Seattle who gave helpful editorial comments.

\section{REFERENCES}

Cauble, R.G., and Mavalwala, J. (1973) The palmar dermatoglyphics of Macaca fascicularis: A comparison with Macaca fuscata. J. Hum. Evol. 2, 137-152.

Iwamoto, M. (1964) Morphological studies of Macaca fuscata. I. Dermatoglyphics of the hand. Primates 5, 53-73.

Iwamoto, M. (1967) Morphological studies of Macaca fuscata. V. Dermatoglyphics of the foot. Primates 8, 155-180.

Iwamoto, M., and Suryobroto B., (1990) Palmar and plantar dermatoglyphics in macaques: A revised method for their description. Primates 31, 431-438. 